\title{
Polymer recycling using microbes
}

\author{
John A. Glaser ${ }^{1}$
}

Published online: 31 July 2017

(C) Springer-Verlag GmbH Germany (outside the USA) 2017

Plastics are globally consumed at a pace of some 311 million tons per year with $90 \%$ having a petroleum origin. Remarkable quantities of packaging plastics are recycled though low. Packaging is the dominating application for these plastics. As an example, beverage bottles constitutes less than $15 \%$ of recycle collection. Generally, plastics degrade in the environment very slowly resulting in major environmental concerns for the proper and sustainable recycling and disposition of such massive amounts of these rather durable organic polymers. There is an increasing collection of technologies and new material sources under development that could provide solutions to current disposal problems of plastics.

A recent discovery of microbial activity capable of complete degradation of widely used beverage bottle plastic expands the range of technology options applicable to plastic disposal. This new research shows that a microorganism isolated from the area adjacent to a plastic bottle-recycling facility can degrade the plastic into small molecular constituents. Poly(ethylene terephthalate) (PET) is a robust organic polymer having a multitude of application ranging from textiles to packaging materials and is the major polymer employed in beverage bottle manufacture. The environmental accumulation of PET is a testament of its versatility and the lack of chemical/physical mechanisms capable of attacking its structural integrity. This new research shows that a newly isolated microbial species, Ideonella sakaiensis 201-F6, degrades PET through hydrolytic transformations by the action of two enzymes, which are extracellular and intracellular hydrolases. A primary hydrolysis reaction intermediate, mono (hydroxy-2-ethyl) terephthalate is formed and can be subsequently degraded to ethylene glycol and terephthalic acid which can be utilized by the microorganism for growth. Environmental Protection Agency.

John A. Glaser

Glaser.John@epa.gov

1 US Environmental Protection Agency, National Risk Management Research, Laboratory, 26 W King Dr, Cincinnati, OH 45268, USA 


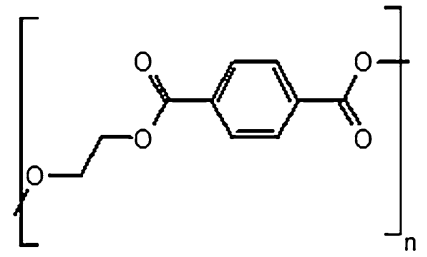

Poly(ethylene terephthalate)
Ideonella sakaiensis

PETase, $\mathrm{H}_{2} \mathrm{O}$

$30^{\circ} \mathrm{C}$



Mono(2-hydroxyethyl) terephthalate

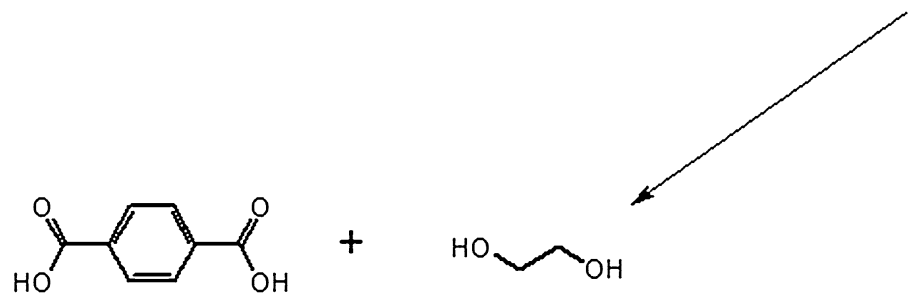

Terephthalic acid

Ethylene glycol


$\mathrm{CO}_{2}$

Microbial conversion of PET to $\mathrm{CO}_{2}$

This discovery could be the beginning of viable technology applicable to the solution of the global plastic problem recognized for its terrestrial component as well as the water contamination problem found in the sea.

Science 2017, 351, 1154-1155; 1196-1199

\section{Change the world}

The editors of Springer Nature journals have assembled groundbreaking scientific findings that have been chosen for their roles to help humanity and protect our planet at a specific web address. The "Change the World, One Article at a Time" initiative draws selected scientific findings and analyses from Springer Nature journals that exemplify research results offering solutions to the global society's most pressing problems. A collection of more than 180 articles spanning areas of interest ranges from Earth and Environmental Sciences to Chemistry, to Physics, Astronomy, Engineering, Materials and beyond. The reader is asked to spread the word about this campaign and share articles with peers and colleagues. The availability of these articles is anticipated to facilitate awareness of research issues and findings. The optional direction of discussion and support can enable developments addressing the challenges of our time leading to opportunities to help to change the world. An example of the literature can be found in the article: Fueling the future: microbial engineering for the production of sustainable biofuels, Nature Revs Microbiol 2016, 14, 288-304. Each of the articles has free access status until the end of July 2017 with open access articles freely available online on a permanent basis.

Nature Geoscience 2016, 9, 161-167; https://www. springernature.com/gp/researchers/campaigns/change-theworld

\section{Cell phone analyzer}

Conventional laboratory science can be taken into the field, the classroom, and the clinic with a host of expanding applications based on a mobile phone. Researchers have used an Apple iPhone 4S with modifications to improve diagnosis and treatment of intestinal worms that infect almost two billion people around the world.

The transformation of a phone to a similar use required a tiny glass lens to form a microscope using the phone's 
camera to magnify objects 50 times. Such a coupling of a smart phone and a multimodal microscope formed a very simple imaging device permitting imaging of DNA sequence reactions. Two battery-powered lasers are used by the microscope to detect different fluorophores and a white LED for bright-field imaging. Several applications show how information gathered by simple field devices can be conducted remotely in the field site leaving the field activity personnel to competently gather data. A study investigating intestinal worms improvised stool sampling by using regular microscope slides, which were covered with cellophane and affixed to the iPhone with double-sided tape. These slides were illuminated with a simple, low-cost flashlight to provide photographs of a magnified image with the phone's camera.

These simple modifications to the phone can be accomplished in a matter of minutes and the supplies are readily available. At the "proof of concept" level of development, this rough assemblage can detect $70 \%$ of the worm infections. Necessary molecular diagnostics for this type of analysis is conducted in well-equipped centralized laboratories often remote to the person experiencing the infection. Clearly, the phone-based device can offer analytical responses to a physician and patient in a greatly shortened timeframe.

This ingenuous way employing cell phones offers a new perspective to promote the development of comparable instruments capable for use as field devices that provide desired/required measurements supporting scientific environmental inquiry. These yet to be invented pocket laboratories can potentially revolutionize data collection and analysis for field investigations and studies with spatial specificity. There are a few private concerns beginning to offer unique instruments utilizing a cell phone as an integral component. With the recognition of the power of such new pocket laboratories, environmental studies and investigations will be changed and possibly expanded to utilize the phone devices to significantly reduce field sampling requiring transport to a distant laboratory. The prospects for this new technology are amazing for the potential ways they can save research resources and be assimilated into field environmental measurements. One notable example of such an application is the use of a smart phone in conjunction with a kite to image the coastline of French Polynesia.

Nature 2017, 545, 119-121; Nature Commun 2017, 8, article number 13913, doi:10.1038/ncomms 13913; The American journal of tropical medicine and hygiene. 2016, 94(1), 158-160

\section{Paperfuge}

The conduct of health care in the third world countries is significantly hampered by the absence of sophisticated analytical/clinical laboratory equipment. Need for analysis is great and often required transportation of a sample to the laboratory capable of conducting the analysis. Recently, a simple device was devised to enable conducting analyses requiring high centrifugal forces such as blood plasma analysis. Requisite commercial centrifuges are costly, large, require electrical power and a motion stable setting. The absence of battery-free point-of-care instrumentation represents a critical block for the development of decentralized treatment. Surprisingly, a simple lightweight, human-powered centrifuge can be assembled from paper. The device has been named "paperfuge." The construction of the paper centrifuge utilizes the design of an ancient whirligig traced to $3300 \mathrm{BC}$. This simple device has been shown to achieve speeds of $125,000 \mathrm{rpm}$ (estimated to be equivalent to centrifugal forces of $30,000 \mathrm{~g}$ ). Speeds of $1,000,000 \mathrm{rpm}$ are theoretically possible with this device. Separation of pure plasma from whole blood was achieved in less than $1.5 \mathrm{~min}$, and malaria parasite isolation was accomplished in $15 \mathrm{~min}$. Similar centrifugal microfluidic devices were constructed from polydimethylsiloxane, plastics, and 3-D polymeric materials. The convenient simple construction opens the development landscape for a multitude of human-powered, cheap, and robust centrifuges for point-of-care use in third world settings and field instruments for the research pursuits of ecology and science education.

Nature Biomed Engin 2017, 1-2. doi: 10.1038/s41551016-0009; 9. doi: 10.1038/s41551-016-0017

\section{Green jet fuel}

The US Department of Energy recent released a 98-page report: Review of Biojet Fuel Conversion Technologies examines the current technologies for producing renewable jet fuels in the categories alcohols-to-jet (ATJ), oil-to-jet (OTJ), syngas-to-jet (STJ), and sugar-to-jet pathways. Each technology development path faces the key challenges of feedstock availability, conceptual process design, process economics, life-cycle assessment of greenhouse gas emissions, and commercial readiness. The main barriers of feedstock price, availability, and energy intensity for each process present significant obstacles to biomass-derived jet fuel. These renewable technologies can potentially replace significant percentages of the fuel requirements for conventional jet fuel necessary to meet commercial and military aviation demand. The aviation industry has selected biomass-derived jet (biojet) as a key element in the strategy to reduce environmental impacts and operating costs. There are demonstrated benefits for the use of biojet fuels related to fuel combustion characteristics and engine tests. Generally, challenges of feedstock availability, conceptual process design, process economics, life-cycle assessment 
of greenhouse gas emissions, and commercial readiness dominate the analysis.

The jet fuel cost, energy supply, energy security, and aviation emissions continue to drive aircraft and engine technology development throughout the history of aviation. Biomass-derived jet fuel offers an opportunity to address sustainability, cost, supply, security, and emissions issues. A range of biomass conversion technologies has been developed to synthesize jet fuels, some of which are commercially viable. The analysis uses four major categories: ATJ, OTJ, GTJ, and STJ to describe each technology with the important factors of feedstocks, upgrading processes, production costs, environmental sustainability and commercial availability. Summaries of production yields, production costs, and GHG emissions for each conversion technology are provided. Not too surprisingly, feedstock was found to be the most important feature when the feasibility of jet fuel production from biomass is considered.

An example of current biomass conversion technology to jet fuel is found with the Honeywell UOP-Honeywell
Green Jet Fuel ${ }^{\mathrm{TM}}$ process. This technology can adjust production of renewable jet fuel or renewable diesel based on market demand and price. The versatile process produces Bio-Derived Synthetic Paraffinic Kerosene or Green Jet Fuel, from hydrogenated esters and fatty acids to meet fuel standards. Input feedstock is flexible for a wide range oils and fats. The process has been evaluated for attractive economics with remarkable payback claiming to reduce costs and risks of achieving high yields.

The Honeywell UOP process is rather flexible in its requirements of oil and fat feedstock and can produce fuels for aviation, marine and highway transportation. This new technology accommodates a wide range of oils and fats as feedstock, and can produce an flexible product slate, to include fuels for aviation, as well as marine and road transportation. It was developed, along with the catalysts and adsorbents, to handle a wide variety of low-cost and contaminated feeds, dramatically improving the economics of the process.

http://www.nrel.gov/docs/fy16osti/66291.pdf
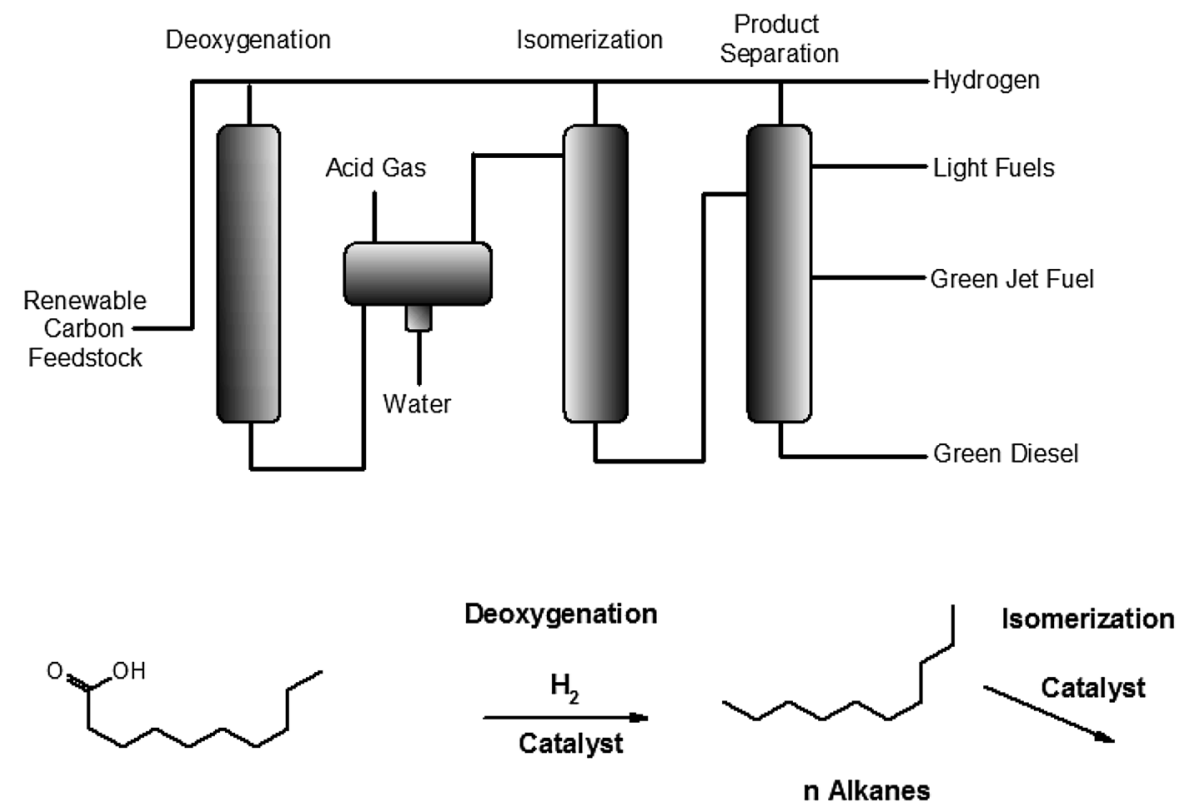

Fatty Acid



Triglyceride

n Alkanes 


\section{Heroes of chemistry awards}

The American Chemical Society sponsors the Heroes of Chemistry annual award to recognize industrial scientists who have achieved the development of products successfully commercialized involving chemistry and having a significant benefit to humankind. GlaxoSmithKline and Shionogi were recognized for their work with dolutegravir, integrase inhibitor for the treatment of HIV-1 infections. Honeywell's development of HFO-1234yf, a non-ozonedepleting refrigerant, was recognized for its contribution as low-global-warming-potential hydrofluoro-olefin refrigerant for mobile air conditioning systems. The new hydrofluoro-olefin refrigerant, 2,3,3,3-tetrafluoropropene (HFO-1234yf) replaces R-134a, a most common refrigerant currently in use, having a global warming potential (GWP) of 1430 compared to a GWP of 4 for the new replacement. The new refrigerant is a "near drop-in replacement" for R-134a, in current air conditioning systems requiring few adaptive changes. Honeywell UOP was recognized for the development and commercialization of Honeywell Green Jet Fuel ${ }^{\mathrm{TM}}$ which is a process technology designed to form jet fuel from waste oils and fats that can be blended seamlessly with commercial petroleum based jet fuel. This new fuel contributes $50-80 \%$ less greenhouse gas based on a life-cycle assessment and can be employed with an existing fueling infrastructure and aircraft engines.

Pfizer was recognized for its commercialization of IBRANCE $^{\circledR}$ (palbociclib) designed for the treatment of metastatic breast cancer (ER-positive and HER2-negative breast cancer). Its mode of action is to act as a selective kinase inhibitor for the cyclin-dependent kinases CDK4 and CDK6. Palbociclib can be combined with other approved hormonal therapies to treat certain patients. The desired selectivity for kinases (CDK) 4 and 6 relative to other cell cycle regulators important for normal human physiology maintenance presented significant technical challenges to the discovery of the target pharmaceutical.

The selection of these technology advances for the award offers an opportunity to inspect the ways in which current industrial pursuits can lead to remarkable opportunities for chemical discovery related to the control of human health-related environmental concerns.

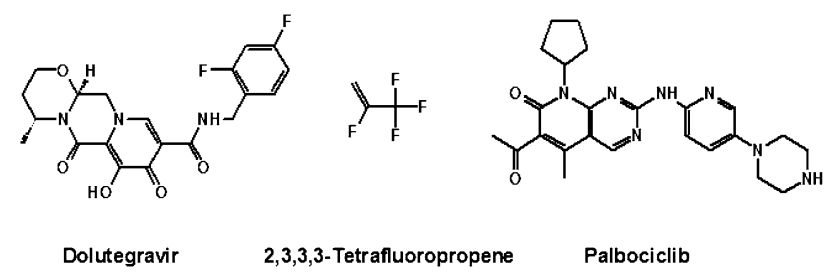

https://www.acs.org/content/acs/en/funding-and-awards/ awards/industry/heroes.html

\section{Nanoreactors}

As we consider the methodology available to accelerate observed reaction rates, the use of increased applied heat, enhanced agitation and other variants but not reaction volume are among the items generally evaluated. Miniature self-assembling "flask" has been explored as a means to accelerate chemical reactions and may be a useful tool in research and industry. These nanoflasks have dimensions of a few nanometers. Confinement volumes comparable to molecular dimensions offer interesting possibilities in the control of the reactive behavior of molecules. The confined spaces of nanostructured materials may offer considerable opportunities for the synthesis of environmentally important chemicals in the future. Constructed materials such as metal organic frameworks, colloidal nanocrystal assemblies, and zeolites are characterized by the slow diffusion of molecules into and out of these materials which has greatly hampered studying the effect of confinement on their physicochemical properties.

Nanoparticle clusters containing empty spaces have been made to self-assemble when exposed to ultraviolet light, and disassemble when exposed to visible light causes leading to potential catalytic cycles for reuse of the nanoflask. By changing certain features of construction, the nanoparticles could be designed to assimilate molecules inside the nanoparticle space (nanoflask) selectively; different nanoflasks can be synthetically designed to accommodate a range of molecular geometries to expand the general utility of enhanced reactivities in nanoparticle space. Different stereochemistries can be easily accommodated in a highly selective manner which could be important to drug synthesis. Visible light illumination causes the nanoflasks to disassemble which releases the product in bulk solution to establish a synthetic catalytic cycle. Such nanoflasks are expected to enable the study of chemical reactivities in confined environments and synthetic approaches to molecules that are difficult to achieve in bulk solution. Reactors can be significantly modified by confinement to match volumes comparable to the dimensions of the molecules. Diffusion limitations can be surmounted by using visible and ultraviolet radiation to create and destroy confined environments. Colloidal nanocrystals functionalized with light-responsive ligands designed to readily self-assemble and trap various molecules from the surrounding bulk solution were used to exploit these 
nanoconditions. The trapped molecules are anticipated to undergo chemical reactions at increased rates and accompanying stereoselectivities remarkably different in bulk solution conditions.

Nature Nanotechnol 2016, 11, 82-88 (2016), p. 6-7

\section{Safer chemical use}

The importance of safety and operational concerns related to chemical operations can be found in chemical hazard assessment methodology organized under the label of GreenScreen ${ }^{\circledR}$ For Safer Chemicals organized by Clean Production Action. The GreenScreen ${ }^{\circledR}$ for Safer Chemicals program is organized as a chemical hazard assessment designed to identify chemicals of high concern and safer alternatives.

It is used by industry, government, and NGOs to support product design and development, materials procurement, and as part of alternatives assessment to meet regulatory requirements. It is used by businesses like Hewlett-Packard, governments like Washington State, and NGOs such as the Healthy Building Network in their Pharos Project. GreenScreen can be used to support environmentally preferable product procurement tools including standards, scorecards, and ecolabels.

Clean Production Action developed an abbreviated version of the GreenScreen method called the GreenScreen List Translator ${ }^{\mathrm{TM}}$, which provides a "list of lists" approach to quickly identify chemicals of high concern. It does this by scoring chemicals based on information from over 40 hazard lists developed by authoritative scientific bodies convened by international, national and state governmental agencies, intergovernmental agencies and NGOs. While List Translator provides an effective way to screen out known hazardous chemicals, an assessment based on GreenScreen ${ }^{\circledR}$ for Safer Chemicals is more comprehensive and can be used to identify safer alternatives.

A new guide entitled How to Use GreenScreen ${ }^{\circledR}$ for LEED $v 4$ is available from Clean Production Action URL which is designed to provide step-by-step instructions to assist industrial personnel to organize a Manufacturer's Inventory, implement the GreenScreen List Translator and/ or Benchmark scores. A pathway is outlined leading to a certification mark, which enables building industry professional's easy recognition of products with this designation. Since the US Green Building Council (USGBC) released LEED v4 in 2013, a strong learning curve for LEED v4 which has been encountered by manufacturers and LEED Practitioners alike who are attempting to be current with the rating system. GreenScreen and
GreenScreen List Translator are claimed to assist this educational concern. GreenScreen ${ }^{\circledR}$ for Safer Chemicals claims to be an open, transparent, and publicly accessible method for chemical hazard assessment which will help to move our society quickly and effectively toward the use of greener and inherently safer chemicals.

https://www.greenscreenchemicals.org/

\section{Green investment selection assistance}

The Church of England has been a strong climate champion in the most recent past years by harassing oil corporations through the support of a global clean energy push. As part of this advocacy, The Church of England has established a climate investment tracker. For a perspective, the Church of England manages $\$ 8.1$ billion in funds. As part of the Transition Pathway Initiative, this new data analysis tool provides to help investors track business risks stemming from the climate considerations. Known as the Transition Pathway Initiative, the tool monitors trends of the four energy-intensive sectors of oil-and-gas, mining, utilities, and vehicles. The initiative will enable the assessment of climate risk as part of investment decision protocol by providing a means to evaluate the progress of companies and inform the investor of a desirable approach for an individual company to take in response to climate challenges. Described as "framework for robust future engagement," the tool is anticipated to aid the continued questioning of the petroleum industry to explain how advances are made to control greenhouse gas emissions to meet 2050 standards.

https://www.churchofengland.org/media-centre/news/ 2017/2017/01/thirteen-leading-international-asset-ownerslaunch-major-initiative-to-embed-climate-concerns-ininvestment-decisions.aspx

\section{Development platform for new antibiotics}

Antibiotic resistance continues as a large assault on the practice of health care and the expectations of the general public who have come to rely on these medicinals for continued preventives necessary to many aspects of health ranging from common bacterial infections to the concerns for infection accompanying any surgery. The rapid evolution of strong resistance in pathogenic bacteria to numerous antibiotics can be traced to the widespread misuse of antibiotics. The loss of effective antibiotics has been intensified through the recent reduced searches for new antibiotics by the pharmaceutical industry. Loss of the 
older reliable antibiotic performers leaves a healthcare gap of potentially apocalyptic dimensions.

A group of researchers has uncovered a remarkable strategy to assist the discovery process for new antibiotic entities. The new chemical synthesis tactics offer a means to access a broad range of potential antibiotics involving a synthetic approach noted for its versatility, modularity, and breadth in terms of different functionalities and topologies. A primary source of antibiotics is compounds occurring in nature that are products of microbial intervention. It is possible to produce such compounds by large-scale fermentation. As medicinal candidates, the natural occurring compounds are hindered by physical characteristics such as bioavailability that can reduce the in vivo activity or metabolic stability. To adjust these constraints, chemical modifications are sometimes required. Due to the complexity of natural products, these modifications must be conducted as a series of steps that permit the modification to be conducted without changing other parts of the starting natural product. Depending on the importance of particular molecular locations, the required chemical modifications may be deemed impractical or in some cases prohibitively difficult for chemical and/or economic reasons. Despite these concerns, semisynthesis is used to access antibiotics for the treatment of various infectious diseases using the chemical modification of structurally complex fermentation products to accomplish the desired transformation. Bacterial resistance has limited the utility of many antibiotics derived from this process.

The authors offer a new synthetic approach to macrolide antibiotics or 14,15 , and 16 membered macrocyclic lactones, which are known for their safe and effective treatment of human infectious diseases such as bacterial pneumonia, gonorrhea and other community-acquired infections. An example of these chemicals is clarithromycin, which can be formed from erythromycin in six semisynthetic steps. The new synthetic pathway uses convergent assembly of simple building blocks to facilitate the synthesis of diverse new candidate antibiotics. Some 300 new candidates are now accessible by this novel synthetic pathway. Evaluation of these new potential antibiotics against a panel of pathogenic microorganisms identified antibiotic activity for the majority of the candidate chemicals, and in some cases efficacious activity toward bacterial strains resistant to current macrolides in use. The chemistry enables the synthesis in a spectacular fashion providing a platform technology sufficiently flexible for continued discovery and is expected to be applicable for manufacturing purposes.

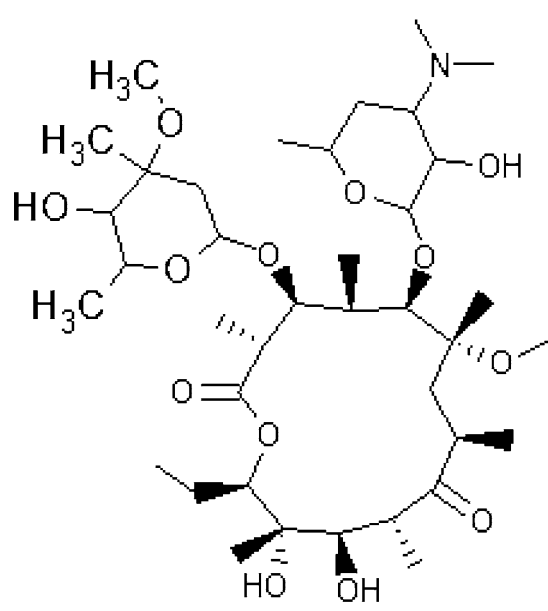

Clarithromycin

Nature 2016, 533, 338-345

\section{New journal}

The new publication, Advanced Sustainable Systems, as an international, peer-reviewed, interdisciplinary journal is designed to offer publishing opportunities for research focused on systems, solutions, technologies and applications and their development and implementation involving the advancement of sustainable living.

Advanced Sustainable Systems strives to connect scientific/technical insights to advances concerning interdisciplinary topics on a wide landscape of topics involving: more efficient and less consumptive technologies, climate engineering, renewable energy, science of air/water/soil issues, sustainable food and agriculture, urban development and sustainable living, ecology, cradle-to-cradle processing, sustainable economics, socio-economic challenges, and policy and governance

Advanced Sustainable Systems will publish articles covering a wide variety of topics from different disciplines that share the focus on the advancement of sustainable living. This will include broad themes such as water and environment, food sustainability, cradle-to-cradle processing, and energy and materials. Publication formats available to Advanced Sustainable Systems authors are: full papers, critical reviews, progress reports, communications, commentaries, viewpoints, research news, and essays.

http://onlinelibrary.wiley.com/doi/10.1002/adsu.v1.1-2/ issuetoc 